\title{
The effects of complexity on the perception of vibrotactile patterns presented to separate fingers
}

\author{
DAVID T. HORNER \\ Indiana University, Bloomington, Indiana
}

\begin{abstract}
Pairs of vibrotactile patterns were presented to subjects' left middle and index fingerpads (unilateral presentation) or left and right index fingerpads (bilateral presentation), using two Optacon arrays. A set of simple (one-line) patterns and a set of complex (two-line) patterns were constructed so that they were equally identifiable when presented individually. In Experiment 1, discrimination performance was lower for two-line patterns than it was for one-line patterns, and it was lower for unilateral presentation than it was for bilateral presentation. Communality, the number of lines that two patterns share in common, was a major factor in reducing discrimination performance for two-line patterns. Subjects' abilities to identify one member of the pair of patterns were measured in Experiment 2. There were no significant differences in performance between pattern sets or type of presentation when subjects attended to a single pattern. However, when subjects were required to attend to both patterns, identification performance was lower for two-line patterns than it was for one-line patterns, and it was lower for unilateral presentation than it was for bilateral presentation. The results suggest that there are limited attentional resources for processing vibrotactile patterns and that more resources are available bilaterally than are available unilaterally.
\end{abstract}

The number of lines in a vibrotactile pattern has been used as one measure of physical complexity. A complex pattern is one that contains more lines than a simple pattern (Craig \& Evans, 1987; Evans, 1987a, 1987b; Evans \& Craig, 1986; Horner, 1991); consistent with this measure of physical complexity is the finding that subjects rate two-line patterns as being higher in perceived complexity than one-line patterns (Horner, 1991). Moreover, although the number of lines in a pattern might be only one of several possible factors that influence perceived complexity, this variable has been shown to have important effects on how vibrotactile patterns are processed (Craig \& Evans, 1987; Evans, 1987a, 1987b; Evans \& Craig, 1986; Horner, 1991). One major effect of adding lines to patterns is to reduce their identifiability (Evans \& Craig, 1986). When Horner (1991) investigated the role of complexity in the perception of patterns presented to a single location-the left index fingerpad-he used a set of one-line patterns and a set of two-line patterns that were equally identifiable, in order to avoid any confounding of differences in complexity with differences in identifiability. When patterns were presented individually, complexity had no effect on performance; identification accuracy and reaction times were similar for one- and two-line patterns (Horner,

This research was supported by Grant DC00095 from the National Institutes of Health. Part of the research was also supported by Northeast Missouri State University. The author wishes to thank James Craig for his comments on this manuscript and Roger Rhodes for programming assistance in the laboratory. Correspondence may be addressed to David T. Horner, Psychology Department, University of Wisconsin Oshkosh, Oshkosh, WI 54901.
1991). However, when pairs of patterns were presented and the time between pattern onsets (stimulus onset asynchrony, or SOA) was varied, discrimination and identification performance were lower for two-line than for oneline patterns (Horner, 1991).

Pairs of patterns presented to the same location at SOAs of less than about 150 msec may be integrated into a single composite representation containing features of both patterns (Evans, 1987a, 1987b; Evans \& Craig, 1986). However, the fact that discrimination performance was lower for two-line than for one-line patterns even at SOAs as long as 400 msec suggests that integration of pattern features was not responsible for the effects of complexity on performance (Horner, 1991). In addition to the temporal separation of pairs of patterns to avoid integrating them into a composite, patterns may be separated spatially by presenting pairs to separate locations. This strategy was adopted in the present study, to determine whether complexity affects performance even when patterns are separated spatially.

Results from previous studies suggest that at brief SOAs, pairs of spatial patterns presented to separate fingers are not temporally integrated in the same manner as are pairs presented to a single finger. For example, Craig (1985a) found that identification performance was reduced when the left and right halves of patterns were presented simultaneously to separate fingers rather than to a single finger, indicating that subjects had difficulty in integrating information from separate fingers. In addition, when judging the temporal order of spatial patterns, subjects may base their decisions on a composite representation when pairs are presented to a single finger, 
but not when pairs are presented to separate fingers (Craig $\& \mathrm{Xu}, 1990)$. Finally, temporal masking declines when pairs of patterns are presented to separate fingers instead of a single finger (Craig, 1983). These and other results indicate that patterns presented successively to a single finger may be affected by factors such as masking and temporal integration, whereas patterns presented to separate fingers may be affected by different factors, such as whether attention can be allocated simultaneously to both fingers (Craig, 1983, 1985a, 1985b).

The ability to process pairs of spatial patterns also deperids on whether they are presented to separate fingers on the same hand or on opposite hands. Discrimination performance is poor when pairs are presented simultaneously to separate fingers on the same hand, and it improves as the SOA is increased beyond about $100 \mathrm{msec}$ (Craig, 1985a, 1985b). However, there is little reduction in discrimination performance at brief SOAs when pairs are presented to fingers on opposite hands, and bilateral performance is superior to unilateral performance (Craig, 1985a). Craig (1985a) also found that performance in identifying spatial patterns split between fingers was better when pattern halves were presented to fingers on opposite hands than when they were presented to fingers on the same hand. These results, showing greater ease in the processing of patterns presented on opposite hands, suggest that greater attentional resources may be available bilaterally than are available unilaterally. It has also been suggested that more resources are required for processing complex patterns than are required for processing simple patterns (Kahneman, 1973). These two suggestions lead to the following prediction: The effect of complexity on the ability to process patterns should be greater when the patterns are presented to fingers on the same hand than when they are presented to fingers on opposite hands. In both of the present experiments, patterns were therefore presented to separate fingers on the same hand and on opposite hands.

In addition to the basic issues of pattern complexity and attentional resources, there is interest in pattern processing at multiple locations, because the use of the hands to explore objects often requires that one combine information from several sites (Craig, 1985a). When the hands move across the surface of an object, the fingers contact different surface features, some of which may be relatively simple, such as an edge or a groove, and some of which may be more complex, such as a raised letter or braille cell. Thus, the tactual identification of an object often involves the processing of spatial patterns of varying complexity that arrive simultaneously, or nearly so, at separate fingers. Some systems of tactual communication rely on the individual's ability to combine complex stimuli from separate fingers. For example, Tadoma (Reed, Durlach, Braida, \& Schultz, 1982) is a method of speech comprehension in which a deaf-blind individual places a hand against a speaker's lips, jaw, and throat to feel facial movements. Information from several fingers must be combined to understand speech. The success of both Tadoma and tactile object recognition (Klatzky, Lederman, \& Metzger, 1985) indicates that, at least to some extent, observers are able to combine information from patterns that arrive simultaneously at several fingers. However, the extent to which pattern complexity affects the ability to combine information from separate fingers has not been explored.

The procedure in the present study was similar to that used previously to determine the effect of complexity on pattern perception (Horner, 1991). A set of one-line patterns and a set of two-line patterns that were equally identifiable were used in discrimination and identification tasks. These two tasks were selected for several reasons. First, the results of these measures can be compared with the results of similar measures that were used in the previous study when patterns were presented to a single finger (Horner, 1991) and in numerous other studies (Bliss, Crane, Link, \& Townsend, 1966; Bliss \& Linvill, 1966; Cholewiak \& Craig, 1984; Craig, 1976, 1983, 1985a, 1985b; Craig \& Evans, 1987; Evans, 1987b; Evans \& Craig, 1986; Horner \& Craig, 1989). Second, the identification and the discrimination of tactile patterns represent two important goals in haptic exploration. In some instances, for example, the tactual exploration of an object involves the identification of different features on the object's surface, and in other instances, a decision is required with respect to whether the features are the same or different; the latter constitutes a discrimination judgment.

\section{EXPERIMENT 1}

Experiment 1 was an examination of whether or not discrimination performance would be similar for pairs of simple and complex patterns presented to separate fingers as a function of the temporal interval between the patterns. Discrimination has sometimes been considered a more sensitive measure of perceptual performance than identification is, because discrimination does not require that one learn names of patterns or search for pattern names stored in memory (Posner \& Mitchell, 1967; Proctor, 1981; Robinson, Brown, \& Hayes, 1964). In previous studies of discrimination at separate sites (Craig, 1985a, 1985b), complexity had not been varied independently of other factors such as set size or identifiability of individual patterns, so it was difficult to make specific predictions about the effect of complexity on the perception of patterns presented to separate fingers. However, as mentioned earlier, discrimination performance should be better bilaterally than unilaterally, and any difference in performance between pattern sets should be in the direction of lower performance for two-line patterns (Horner, 1991).

Communality has been shown to have a potentially large effect on discrimination performance (Geldard \& Sherrick, 1965; Gilson, 1968; Gottheil, Cholewiak, \& Sherrick, 1978). The term communality refers to the number of elements that are common to a pair of patterns; pat- 
terns with more elements in common are less discriminable. Because pairs of patterns had been presented to a single location in previous studies of discrimination, elements common to each pattern in the pair stimulated identical positions on the skin. For example, the vertical lines in the patterns $L$ and $F$ would stimulate identical positions on the same fingerpad. However, when these patterns are presented to separate fingers, the vertical lines in each pattern obviously do not stimulate identical skin positions; the lines are "common" to the pair of patterns only in the sense that they stimulate homologous positions on each fingerpad. Presenting patterns to separate sites affords the opportunity to address the issue of whether the effects of communality on discrimination performance depend on elements stimulating identical skin positions, or whether communality has its effects at a later stage of processing when representations of patterns are being compared. Is a line common to a pair of patterns only when the line stimulates identical skin positions, or is it a common line by virtue of its spatial relationship to other lines in the patterns? Horner (1991) showed that there was a large effect of communality on the ability to discriminate oneand two-line patterns presented to a single fingerpad. Although the one-line patterns used in the present study contained no lines in common (see Figure 1), some of the two-line patterns contained a common line, and these differences in communality may affect discrimination performance.

\section{Method}

Subjects. Six subjects were tested in the main portion of the experiment. All had received practice in identifying letter-like patterns and one- and two-line patterns. Five undergraduate women
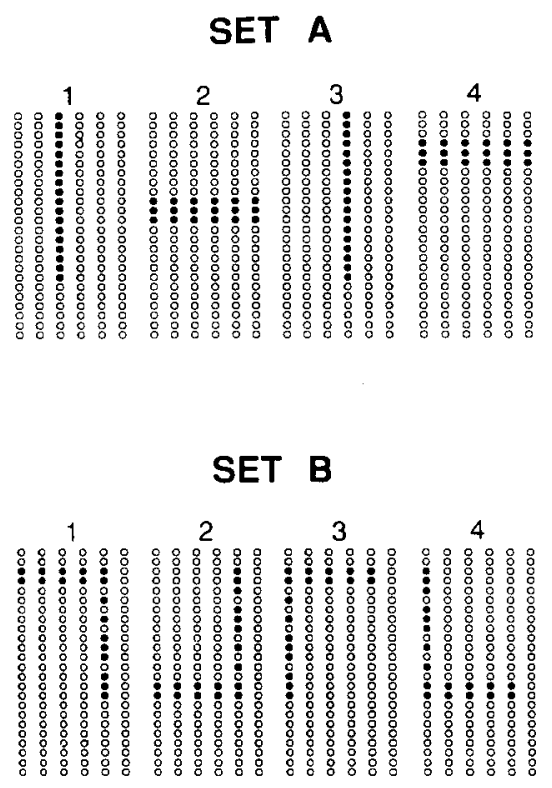

Figure 1. Illustrations of the two pattern sets used in the experiments. Patterns in Set $A$ are composed of single lines, and patterns in Set $B$ are composed of two lines. were paid an hourly wage for participating in the experiment. The experimenter was the 6th subject, and his data were included in the analysis of the results.

Apparatus. The apparatus consisted of two vibratory arrays from the Optacon (Bliss, Katcher, Rogers, \& Shepard, 1970) interfaced with a PDP-11/34 computer. Each array measured $1.1 \times 2.7 \mathrm{~cm}$ and consisted of 144 blunt pins arranged in 24 rows and 6 columns. The computer controlled the amplitude and duration of vibration of the individual pins. Each of the pins vibrated at $230 \mathrm{~Hz}$, and the intensity of the vibration was set at a comfortable level, $33 \mathrm{~V}$ to the driver circuits. Each vibratory array was placed in contact with the distal portion of a separate fingerpad. Further details about the tactile arrays may be found in Craig (1980).

Stimuli. The two sets of patterns from Experiments 1 and 2 are shown in Figure 1. A pilot experiment was conducted to select a set of one-line patterns and a set of two-line patterns that were equally identifiable - that is, so that the percentages of correct responses for both pattern sets were nearly identical. Set A consisted of four patterns that each contained a single line; horizontal lines consisted of three adjacent rows of pins, and vertical lines consisted of the top (distal) 18 pins in a single column. The subjects did not feel individual pins separately. So, for example, when Stimulus 2 was presented, the subjects reported feeling a single horizontal line on the fingerpad. Set B consisted of four patterns that each contained two lines. The gaps in the vertical lines caused by leaving certain pins inactivated were not perceived by the subjects as gaps. These gaps were necessary to equate the number of pins in each pattern. As can be seen in Figure 1, the patterns in both sets contained the same number of pins, 18. The term line is used for ease of discussion and is not meant to imply that the visual and tactual perceptions of these patterns were similar.

The position of each line in a two-line pattern was duplicated in other two-line patterns so that the subjects could not identify a twoline pattern by locating only a single line. The patterns contained no active pins in the top two or bottom eight rows of the array, and thus the patterns were presented within an area on the fingerpad approximately $1.1 \times 1.5 \mathrm{~cm}$. The patterns were presented for $26 \mathrm{msec}$ in a "static" mode (Craig, 1980), in which all of the pins in the pattern were activated simultaneously.

Procedure. The patterns in Figure 1 were originally members of larger one- and two-line pattern sets (eight patterns per set) used in a previous study (Horner, 1991). The subjects were asked to judge the complexity of each of the patterns in these eight-pattern sets. Five subjects with several months of laboratory experience at feeling vibrotactile patterns were asked to rate the complexity of each pattern on a 9-point scale, with 9 representing a judgment of very complex. The mean complexity ratings for the patterns in Figure 1 showed that the subjects judged two-line patterns (3.82) to be more complex than one-line patterns (1.97). A group of 6 inexperienced subjects who had never felt vibrotactile patterns were also tested, and they judged two-line patterns (4.58) to be more complex than one-line patterns (2.91). Although the subjects were allowed to use ratings from 1 to 9 , they did not use the entire range of values: The minimum complexity rating for each one-line pattern averaged across all subjects was 1.59 , and the average maximum rating for each two-line pattern was 6.47 .

In the discrimination task, which made up the main portion of the experiment, both pattern sets in Figure 1 were tested separately within a session, and the order in which the two pattern sets were tested alternated from session to session. The subjects were seated with two fingers on the tactile arrays in front of them, one array per finger. For unilateral presentation, the subjects placed their left middle and index fingerpads on the arrays and responded with their right hands by pressing the appropriate key on a computer keyboard. For bilateral presentation, the subjects placed their left and right index fingerpads on the arrays and responded by using footpedals that were also interfaced with the computer. The subjects wore ear- 
phones through which low-pass, filtered noise was fed to mask auditory stimuli from the array.

On each trial, the subjects received a cue stimulus consisting of the topmost left and topmost right pins in each array, which were simultaneously activated for $13 \mathrm{msec}$. The cue stimuli also served as a way to ensure that each finger was correctly positioned on each array. One second after the cue stimuli, a pattern was presented to each fingerpad. The patterns were presented simultaneously (an SOA of $0 \mathrm{msec}$ ), and at SOAs of 26, 52, 100, and $400 \mathrm{msec}$. The first pattern was always presented to the left middle finger for unilateral presentation and to the left index finger for bilateral presentation. The subjects judged whether the patterns in the pair were the same or different (an " $S$ " response or a " $D$ " response, respectively) after the pair was presented. The subjects were told that on half of the trials a pair of identical patterns would be presented (a same trial) and on half of the trials a pair of different patterns would be presented (a different trial). Trial-by-trial feedback was provided, consisting of either the word "correct" appearing on a visual display in front of the subject, or, when the response was incorrect, an " $S$ " or a " $D$ " corresponding to the correct choice. Unilateral and bilateral presentations were tested on alternate days. Within an experimental session, same and different trials were presented randomly in blocks of 30 , with each randomly presented block used to test a different SOA.

In addition to the discrimination measurements, identification performance was measured. Patterns were presented individually to each of the three fingers-left middle, left index, and right index-to ensure that one- and two-line patterns were equally identifiable under all conditions, and also to ensure that any differences between bilateral and unilateral performance would not be due to differences in sensitivity among the three fingers.

\section{Results and Discussion}

The percentage of correct identification responses averaged across all subjects differed for one- and two-line patterns by only $1 \%$ on each finger. Identification performance averaged across pattern sets and subjects was $96 \%$ correct on the left index and left middle fingers, and $91 \%$ correct on the right index finger. Lower identification performance on the right index finger may have been due to the fact that subjects seldom use the right hand for testing in the laboratory, and thus the right index finger may have received less practice in feeling vibrotactile patterns than did fingers on the left hand. Identification perfor-

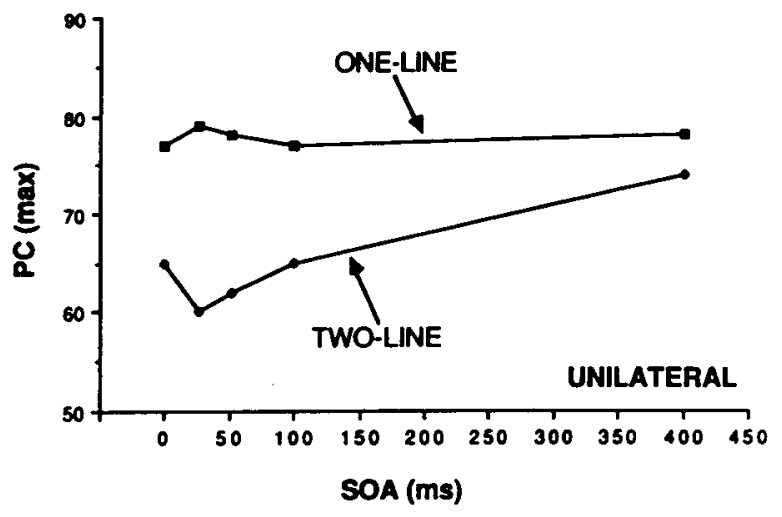

mances for individual subjects were similar to the averages above.

The results from the discrimination task were used to calculate $P(\mathrm{C}) \max$ at each SOA. This statistic (McFadden, 1970), used in previous studies of discrimination (Cholewiak \& Craig, 1984; Craig, 1983; Horner, 1991; Horner \& Craig, 1989), minimizes effects of response bias. The subjects responded " $S$ " more often than expected by chance at the briefest SOAs $(0-100 \mathrm{msec})$, and the average rates of responding " $S$ " ranged from $54 \%$ to $61 \%$ in the unilateral condition and from $51 \%$ to $54 \%$ in the bilateral condition. There were no systematic differences in response bias for the one- and two-line patterns. The data were scored in terms of hits (responding " $D$ " on a different trial) and false alarms (responding "D" on a same trial). Hits and false alarms were used to calculate $d^{\prime}$ and $P(C) \max$. The use of $P(\mathrm{C}) \max$ changed the percentage of correct responses by less than $3 \%$. $P(\mathrm{C})$ max is plotted as a function of SOA for both the unilateral and the bilateral conditions in Figure 2. All data points represent the mean of 1,080 trials, 180 trials for each of the 6 subjects. The standard errors of the means ranged from $2 \%$ to $3 \%$ for each pattern set and did not differ systematically for unilateral and bilateral presentation. The data for each subject were similar to those shown in Figure 2.

The functions in Figure 2 clearly show that discrimination performance was higher for one-line than for twoline patterns for both unilateral and bilateral presentation. The results of a three-way, repeated measures analysis of variance revealed effects of pattern set $[F(1,5)=$ $25.31, p<.01]$, presentation type (unilateral vs. bilateral) $[F(1,5)=24.14, p<.01]$, and SOA $[F(4,20)=4.80$, $p<.01]$. The effect of presentation type reflects the fact that discrimination performance was better when patterns were presented to fingers on opposite hands than when they were presented to fingers on the same hand, and this replicates the finding that it is easier to process patterns presented bilaterally than it is to process patterns presented unilaterally (Craig, 1985a). The effect of SOA reflects

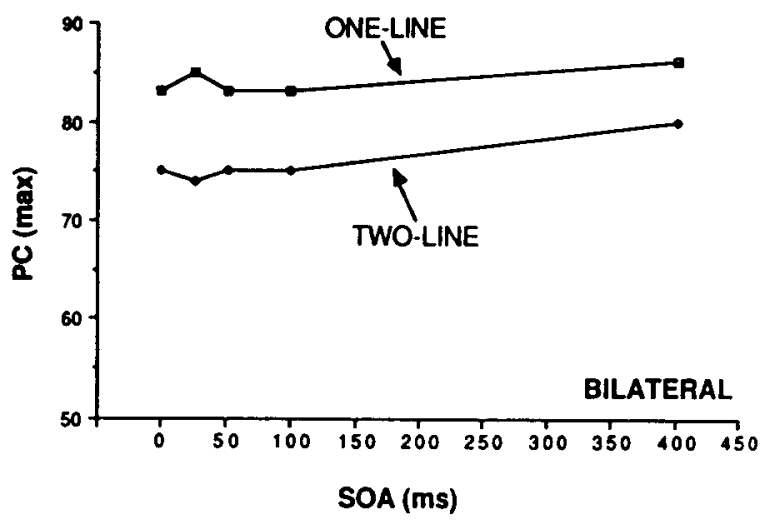

Figure 2. Percent correct discrimination responses, $P(\mathbf{C}) \mathbf{m a x}$, as a function of stimulus onset asynchrony for unilateral and bilateral presentation. The upper function in each graph represents performance for one-line patterns, and the lower function represents performance for two-line patterns. 
the overall shapes of the functions in Figure 2: discrimination performance generally improved as the SOA was increased. None of the interactions were significant ( $p>.05$ in all cases) except for that between pattern set and SOA $[F(4,20)=4.3, p<.01]$. This interaction indicates that the difference in performance between oneand two-line patterns was larger at brief SOAs, particularly for unilateral presentation.

The fact that there was no interaction between type of presentation and pattern set might be taken to indicate that the difference in performance between pattern sets was about equal for bilateral and unilateral presentation. However, as noted above, the interaction between pattern set and SOA indicated that the difference in performance between pattern sets varied as a function of SOA and was larger at brief SOAs. To determine whether the difference in performance between pattern sets at brief SOAs was larger for unilateral than for bilateral presentation, performance for each pattern set was averaged across the briefer SOAs $(0-100 \mathrm{msec})$. At brief SOAs, the difference in average performance between pattern sets was $14 \%$ for unilateral presentation and only $8 \%$ for bilateral presentation. These results indicate that the effect of complexity on discrimination performance at brief SOAs is greater when patterns are presented unilaterally than it is when they are presented bilaterally.

Horner (1991) showed that the variation in the ability to discriminate patterns presented to a single location depended on whether pairs of different two-line patterns shared a line in common or shared no lines in common. When two-line patterns were chosen for the present study, the positions of the lines were duplicated so that the subjects could not identify patterns by locating only a single line. Because of this duplication, some different pairs contained one line sharing the same position (50\% communality), and other different pairs shared no lines (0\% communality). For example, in Set B of Figure 1, Patterns 3 and 4 share a line in common, and Patterns 1 and 4 share no lines in common. The results were therefore analyzed to determine whether communality affected performance

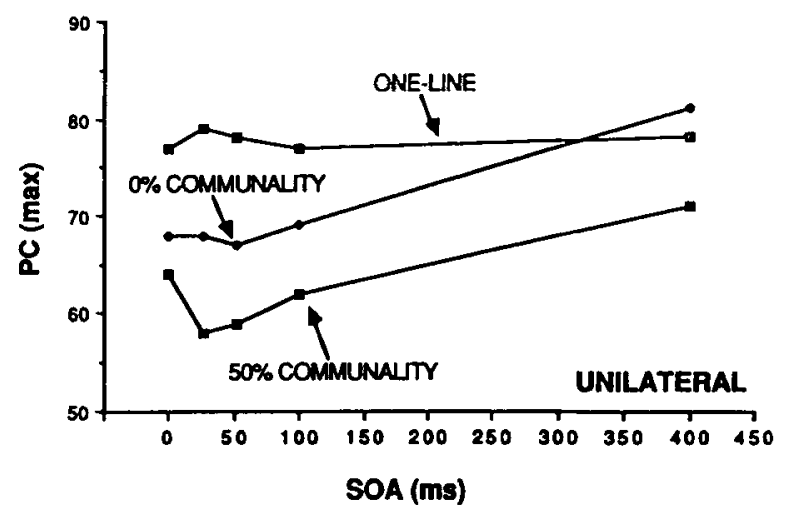

when patterns were presented to separate fingers. (For a description of the communality analysis, see Horner, 1991, Experiment 1.) $P(C) \max$ functions for two-line patterns with $0 \%$ and $50 \%$ communality, as well as the one-line $P(\mathrm{C}) \max$ functions from Figure 2, are shown in Figure 3 for both the unilateral and the bilateral conditions.

Figure 3 shows that at all SOAs, performance was lower for patterns with $50 \%$ communality than for patterns with $0 \%$ communality, indicating that communality played a major role in the ability to process patterns presented to separate fingers. The separation between the $0 \%$ and $50 \%$ communality functions resembles the effect of communality when pairs of patterns are presented successively to the same finger (Horner, 1991). The fact that an increase in communality reduces discrimination performance regardless of whether pairs of patterns are presented to the same location or to separate locations indicates that the effects of communality do not depend on pattern elements' stimulating identical skin positions. The results suggest that a line may be common to a pair of patterns solely by stimulating homologous positions on separate fingers. However, the results in Figure 3 differ for unilateral and bilateral presentation. Looking first at the unilateral results, performance at brief SOAs was lower for two-line patterns at both levels of communality than for one-line patterns, indicating that regardless of level of communality, attending simultaneously to both fingers was more difficult with two-line than it was with one-line patterns. At the longest SOA, performance was similar for one-line patterns and two-line patterns with $0 \%$ communality. The bilateral results showed that performance was similar for one-line patterns and two-line patterns with $0 \%$ communality at all SOAs. Thus, the main reason for the difference in performance between pattern sets in the bilateral condition appeared to be difficulty in discriminating pairs of two-line patterns that shared a line in common.

The fact that one- and two-line patterns presented individually were equally identifiable (in the present experi-

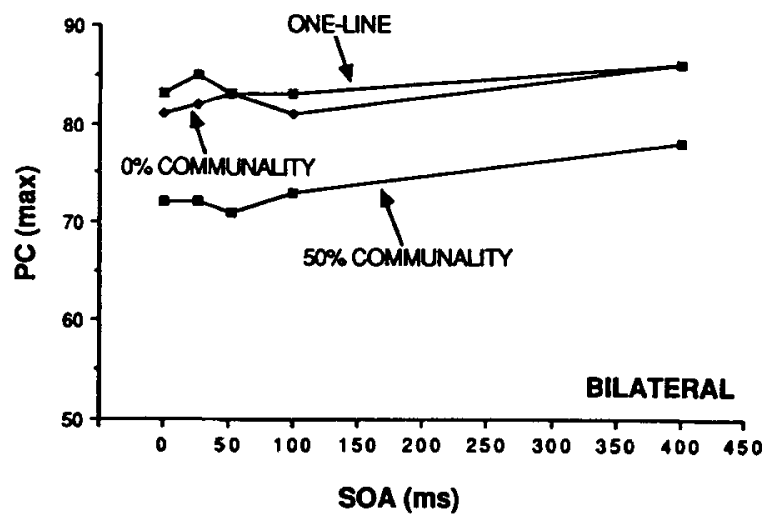

Figure 3. The data from Figure 2, replotted to show percent correct discrimination responses, $P(C)$ max, for one-line patterns, and $P(C)$ max for pairs of different two-line patterns that share one line in common ( $50 \%$ communality) or no lines in common (0\% communality). Performance is shown as a function of stimulus onset asynchrony for the unilateral and bilateral conditions separately. 
ment and in Horner, 1991) and elicited similar reaction times (Horner, 1991) suggests that patterns judged to be simpler or more complex may nonetheless be processed with equal efficiency, at least according to these measures. However, when pairs were presented to separate fingers (in the present experiment) or to the same finger (Horner, 1991), complex patterns were less discriminable than simple patterns were. Allocating limited attentional resources to pairs presented close together in time may cause poorer performance for complex than for simple patterns if complex patterns require more resources (Kahneman, 1973). As the time between pairs is increased, resources may be allocated first to one pattern and then to the other, and the difference in performance between simple and complex patterns may decline. The fact that the effect of complexity on performance was reduced when pairs were presented to fingers on opposite hands suggests that more resources may be available for processing patterns bilaterally than for processing them unilaterally.

Why should greater resources be required for discriminating two-line patterns than for discriminating one-line patterns when they are equally identifiable? One possibility is that separate factors limit the discrimination of one- and two-line patterns. For example, discrimination of one-line patterns may be limited by the ability to localize each pattern on the fingerpad, whereas discrimination of two-line patterns may be limited by the ability to compare relationships between lines within each pattern, a process that might require greater attentional resources than would localization. An analysis of confusion data from the identification task indicated that lines with the same orientation on the fingerpad were clearly discriminable, but were sometimes confused with one another. Comparing the confusion data for one- and two-line patterns is uninformative because of the nature of the patterns used in the present study; two-line patterns share common lines, and one-line patterns have no lines in common. To address this issue further, one could construct sets of oneand two-line patterns that were equally identifiable and had similar types of confusions, and then examine whether they were equally discriminable.

\section{EXPERIMENT 2}

Does complexity affect performance only when one is processing both patterns in a pair, or does the mere presence of an additional pattern result in lower performance for complex than for simple patterns? Horner (1991) presented either one- or two-line target patterns and masking stimuli successively to a single location and found that identification performance was lower for two-line than for one-line patterns, thus showing that complexity affects performance even when subjects are not required to process both patterns in a pair. However, integration of target and masker features over brief temporal intervals might have prevented subjects from processing targets independently.

In Experiment 2, pairs of patterns were presented to separate fingers and subjects identified only one pattern.
In the directed-attention condition, the subjects were told before each trial which pattern to identify. In the dividedattention condition, the subjects were told which pattern to identify only after both patterns were presented. One might expect similar performance for one- and two-line patterns in the directed-attention task because little masking occurs when patterns are presented to separate fingers, and this task would be similar to the single-pattern identification task in Experiment 1. On the contrary, the discrimination results of Experiment 1 suggest that performance in the divided-attention condition should be lower for two-line than for one-line patterns, and the difference in performance should be greater for unilateral than for bilateral presentation. Previous results (those of the present Experiment 1 and those in Craig, 1985a, 1985b) also predict that performance should be lower in the divided-attention than in the directed-attention condition and that performance should be higher for bilateral than for unilateral presentation.

\section{Method}

Subjects. Six undergraduate women, 4 of whom had participated in Experiment 1, were paid an hourly wage to serve as subjects. Apparatus. The same apparatus was used as in Experiment 1.

Procedure. The same stimuli and testing procedures were used as in Experiment 1, except that instead of discriminating the pair of patterns, subjects identified one member of the pair. The four patterns in the set currently being tested were illustrated on a card placed in front of the subjects and numbered as in Figure 1. The subjects were informed by means of the visual display which member of the pair to identify. In the directed-attention condition, they were told which pattern to identify before each trial began. In the dividedattention condition, they were told which pattern to identify $500 \mathrm{msec}$ after both patterns had been presented. In the dividedattention condition, the same SOAs were tested as in the discrimination task. The pattern that the subjects were told to identify was randomly selected on each trial. They received trial-by-trial visual feedback, which consisted of either the word "correct," or, when the response was incorrect, the number of the correct pattern. Trials were presented in blocks of 25 (bilateral) or 30 (unilateral) and each randomly presented block was used to test a different SOA. Smaller blocks of trials were necessary for bilateral presentation, because it took longer to respond with foot pedals. Performance in identifying patterns presented individually was not measured.

\section{Results and Discussion}

The mean results across all subjects are shown in Figure 4 for both the unilateral and the bilateral conditions. Percent correct identification for each pattern set is shown for the directed-and divided-attention conditions as a function of SOA. For the unilateral results, each data point represents the mean of 1,080 trials, 180 trials for each of the 6 subjects. For the bilateral results, each data point represents the mean of 1,050 trials, 175 trials for each subject. The standard errors of the means ranged from $2 \%$ to $3 \%$ for each pattern set and did not differ systematically for unilateral as opposed to bilateral presentation. The data for each subject were similar to the mean results shown in Figure 4.

Performance was lower in the divided-attention condition than in the directed-attention condition for both unilateral and bilateral presentation. However, varying 

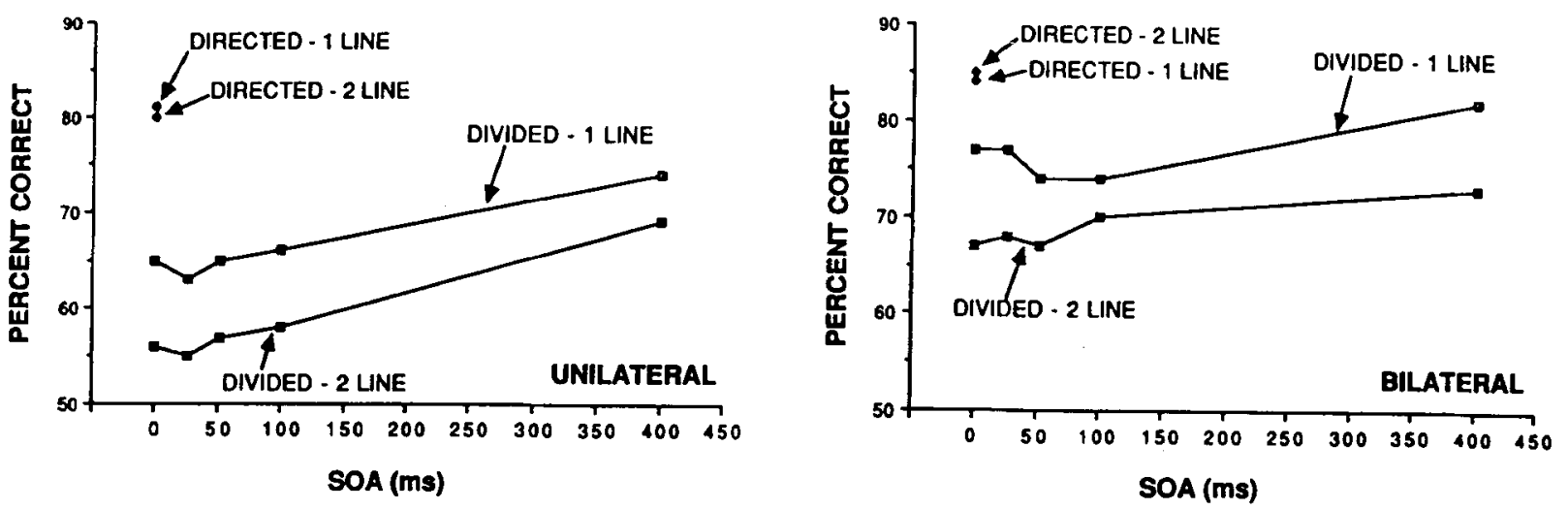

Figure 4. Percent correct identification responses as a function of stimulus onset asynchrony for unilateral and bilateral presentation. The two data points in the upper left of each graph represent directed-attention performance for one- and two-line patterns. The upper functions in each graph represent divided-attention performance for one-line patterns, and the lower functions represent divided-attention performance for two-line patterns.

the number of lines in a pattern had a much different effect on directed-attention performance than on dividedattention performance. In the directed-attention condition, performance was nearly identical for one- and two-line patterns for both unilateral and bilateral presentation. This result is not surprising, since one- and two-line patterns were nearly equally identifiable when presented individually in Experiment 1. The data from the directedattention condition were subjected to a two-way, repeated measures analysis of variance, which revealed no effect of pattern set $[F(1,5)=0.01, p>.91]$, no effect of presentation type (unilateral vs. bilateral) $[F(1,5)=3.79$, $p>.11]$, and no interaction $[F(1,5)=3.97, p>.10]$. Directed-attention performance was unaffected by whether patterns were presented to fingers on the same hand or on opposite hands and was also unaffected by the pattern set tested.

The data from the divided-attention condition were subjected to a three-way, repeated measures analysis of variance, which revealed an effect of presentation type $[F(1,5)=11.45, p<.02]$ and an effect of SOA $[F(4,20)=$ $13.67, p<.01]$. As was true for discrimination performance, identification performance generally improved as the SOA was increased, and it was higher for bilateral than for unilateral presentation. However, there was no effect of pattern set $[F(1,5)=6.52, p>.05]$, even though performance in the divided-attention condition was lower for two-line than for one-line patterns. There were no interactions ( $p>.05$ in all cases).

The lack of an effect of pattern set for the dividedattention data seems surprising in light of Figure 4 and the discrimination results of Experiment 1. An analysis of individual subjects' data revealed some small differences between directed-attention performance for the two pattern sets, and one of the subjects showed slightly better divided-attention performance for two-line than for one-line patterns, even though the difference between directed- and divided-attention performance was larger for two-line than for one-line patterns. Because of these differences in individual subjects' data, and to address directly the question of differences in subjects' abilities to process patterns in the two conditions, directed-attention performance was used as a baseline for comparison with divided-attention performance. For each subject's data, divided-attention performance at each SOA was subtracted from directed-attention performance, and the average results across subjects are shown in Figure 5. These data were subjected to a three-way, repeated measures analysis of variance, which revealed effects of pattern set $[F(1,5)=21.68, p<.01]$ and SOA $[F(4,20)=13.67$, $p<.01]$, but no effect of presentation type $[F(1,5)=$ $4.03, p>.10]$. There were no interactions $(p>.05$ in all cases). These data clearly show that in relation to directed-attention performance, divided-attention performance showed a larger decline for two-line than for oneline patterns.

What might be responsible for the greater decline in divided-attention performance for two-line patterns than for one-line patterns? One tempting explanation is that pairs of two-line patterns mask one another to a greater degree than do pairs of one-line patterns. However, as mentioned previously, there is little masking between separate fingers. Furthermore, the fact that there was no difference between pattern sets in directed-attention performance suggests that if pairs of patterns did mask one another, levels of masking were the same for one- and two-line patterns. Craig (1983, 1985a) has investigated the possibility that masking plays a major role in the results of directed-/divided-attention tasks and has argued that reduced divided-attention performance at brief SOAs is not due to masking.

The difference in divided-attention performance between pattern sets is consistent with the view expressed earlier that limited attentional resources must be allocated to separate fingers. There would be more errors in naming two-line patterns than there would in naming one-line 

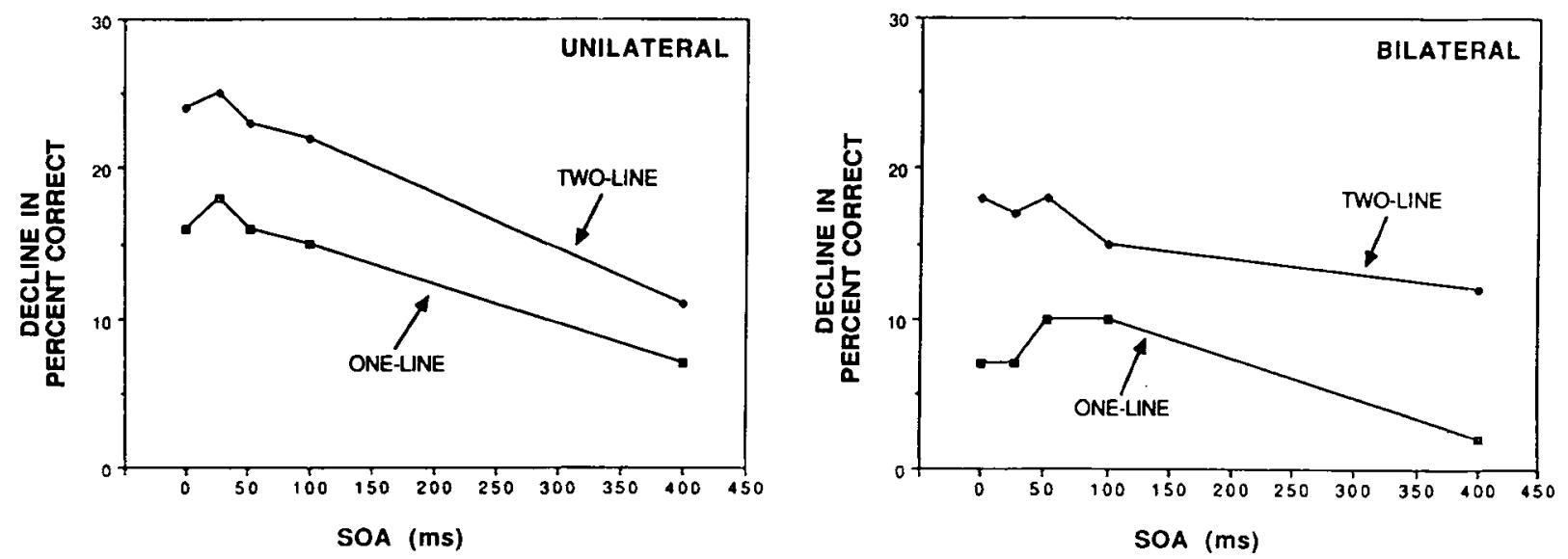

Figure 5. The data from Figure 4, replotted to show the decline in divided-attention performance (percent correct) compared with directedattention performance at each stimulus onset asynchrony for unilateral and bilateral presentation. The upper functions in each graph represent a larger decline in performance for two-line patterns, and the lower functions represent a smaller decline in performance for one-line patterns.

patterns if two-line patterns required more resources (Kahneman, 1973). In the directed-attention condition, there may be sufficient resources to name one- and twoline patterns with equal efficiency. Evans and Craig (1991) have shown that subjects may not be able to restrict processing to a single finger, and that some resources may be devoted to processing stimuli on adjacent fingers. An analysis of the directed-attention data showed that subjects' incorrect responses were most often the nontarget member of the pair, suggesting that subjects were unable to restrict processing to a single finger. Furthermore, the fact that this occurred for both one- and two-line patterns indicates that, regardless of complexity, on at least some of the trials the nontarget pattern may be processed to a relatively late stage.

Previous bilateral presentation results (Craig, 1985a) have shown a smaller difference between directed- and divided-attention performance than was found in the present study. However, the fact that different patterns were used in the two studies may explain the discrepancy in results. Craig used a set of 10 patterns that were identified correctly about $96 \%$ of the time in the directedattention condition, whereas directed-attention performance was only about $85 \%$ for the patterns used in the present study. The ability to process pairs of patterns presented to separate fingers might improve when the patterns are easier to identify. In addition, the patterns that Craig used contained some redundancy, so that each pattern could have been identified through the processing of only a part of the pattern, whereas the patterns used in the present study required the processing of the entire pattern. Furthermore, identifying one- and two-line patterns requires that one localize individual lines, potentially a strategy much different from that used to identify the 10 patterns in Craig's study.

Divided-attention performance improved as the SOA was increased, and Craig (1985a, 1985b) has suggested that subjects may use an increased temporal separation to switch attention between fingers, resulting in improved performance. The fact that performance for two-line patterns remains below that for one-line patterns even at SOAs as long as 400 msec suggests that it may take longer to switch attention for more complex patterns. This conclusion must remain tentative, however, because performance did not increase to the level of directed-attention performance. Hence, the entire shape of the function relating percent correct to the time between patterns cannot be compared for simple and complex patterns.

Experiment 1 showed that the difference in discrimination performance between pattern sets at brief SOAs was larger for unilateral than for bilateral presentation. To determine whether the same would be true for the identification results, divided-attention performance for each pattern set was averaged across the briefer SOAs $(0$ $100 \mathrm{msec}$ ). Unlike the discrimination results, the difference in average performance at brief SOAs between pattern sets was the same, about $8 \%$ for both unilateral and bilateral presentation. However, unilateral divided-attention performance for two-line patterns was near chance (the chance level of performance was estimated by assuming that subjects were able to attend to only one finger; see Craig, 1985a), and this may have limited the size of the difference in performance between one- and two-line patterns.

\section{GENERAL DISCUSSION}

There were four main results of the present study: (1) an increase in pattern complexity reduced the ability to process both members of a pair of patterns presented to separate fingers, (2) overall performance was better when patterns were presented to fingers on opposite hands than when they were presented to fingers on the same hand, (3) the effect of complexity on discrimination performance 
at brief SOAs was greater for unilateral than for bilateral presentation, and (4) at longer SOAs, overall performance improved for both one- and two-line patterns.

These results can be accomodated by the notion of a limited amount of attentional resources for processing vibrotactile patterns. Such limited-resource models of attention are common (Kahneman, 1973; Knowles, 1963; Moray, 1967; Navon \& Gopher, 1979; Norman \& Bobrow, 1975). Specifically, the effects of complexity on performance in the discrimination and divided-attention tasks of the present study may have been due to limited attentional resources allocated to pairs of patterns presented simultaneously to separate fingers. Complex patterns may require more resources than simple patterns require, and allocating resources to pairs of patterns may reduce performance more for complex patterns than for simple patterns. In the directed-attention condition, subjects may devote all of the available resources to processing a single pattern, and performance is about equal for simple and complex patterns.

One- and two-line patterns presented individually were equally identifiable, indicating that sufficient resources were available for processing one- and two-line patterns with equal efficiency (see also Horner, 1991). Nonetheless, more resources may be required for identifying twoline patterns than are required for identifying one-line patterns. The fact that two-line patterns were rated higher in perceived complexity than one-line patterns were, even when identification performance was about equal for the two pattern sets, may reflect an intuitive notion on the part of subjects that two-line patterns require more effort to process than one-line patterns do. The idea that complex patterns require more resources than simple patterns do might be investigated further by using dual-task techniques in which subjects maximize performance on a primary task while also performing a secondary task (Navon \& Gopher, 1979; Posner, 1978, 1982; Wickens, 1984). Performance on the secondary task is a measure of the excess resources not devoted to the primary task. For example, subjects might perform a primary task of identifying individual one- or two-line patterns, and some secondary task as well. If one- and two-line patterns were equally identifiable, performance on the secondary task would be a measure of the amount of excess resources available. Because there would be fewer excess resources available when subjects were identifying two-line patterns, performance on the secondary task would be lower when the task was combined with identifying two-line patterns than when it was combined with identifying one-line patterns.

The results of the present study also support previous evidence that vibrotactile spatial patterns are easier to process when they are presented to fingers on opposite hands than when they are presented to fingers on the same hand (Craig, 1985a; Craig, Green, \& Rhodes, 1985; Lappin \& Foulke, 1973). Discrimination and divided-attention performance were higher when pairs of patterns were pre- sented bilaterally than when they were presented unilaterally. Furthermore, the effect of complexity on performance at brief SOAs was reduced when patterns were presented to fingers on opposite hands. These results suggest that there may be greater resources for processing patterns bilaterally than there are for processing them unilaterally.

Whether attentional resources can be allocated simultaneously to separate locations without a loss in performance may also depend on overall task demands. In a detection task, for example, when subjects have to attend to several widely spaced body locations, there is no difference in performance whether stimuli are presented simultaneously or successively (Shiffrin, Craig, \& Cohen, 1973). This result indicates that sufficient attentional resources are available for the detection of vibrotactile stimuli. However, in the present study, identification and discrimination performance were reduced when pairs of spatial patterns were presented to separate locations at brief SOAs, indicating that insufficient resources were available for these more demanding tasks.

In addition to theoretical issues of complexity and resource allocation, the results of the present study have implications for the use of the skin as a channel of communication for deaf or blind individuals. One implication is that increasing the complexity of spatial patterns that are used in systems of tactual communication may lead to difficulty in processing patterns that are presented successively to separate locations. When spatial patterns are presented successively to a single location, temporal masking is often a major factor limiting perception (Cholewiak \& Craig, 1984; Craig, 1976, 1978, 1982, 1983, 1985b; Craig \& Evans, 1987; Evans \& Craig, 1986; Horner \& Craig, 1989), and it may reduce the rate at which patterns may be presented with tactual communication devices such as the Optacon and braille (Nolan \& Kederis, 1969). Moreover, temporal masking is greater for complex than for simple patterns when stimuli are presented successively to a single location (Horner, 1991). One suggestion for avoiding the limitations imposed by temporal masking has been to present patterns to separate locations, such as two different fingers (Craig, 1985b). The problem with this strategy appears to be that it is difficult to attend simultaneously to separate fingers (Craig, 1985a, $1985 \mathrm{~b}$ ). In addition, the results of the present study indicate that complexity has similar effects on pattern perception, whether patterns are presented successively to the same location or to separate locations. If several locations are used for presenting complex spatial patterns, the time between patterns may have to be extended to allow acceptable levels of performance. This in turn may reduce the rate at which complex patterns may be presented.

Although an increase in pattern complexity is likely to reduce rates of processing in tactual communication systems, there are other reasons why increasing complexity might not produce extremely slow rates. First, context 
cues are often available, as in the reading of printed text with the Optacon, or in reading braille. Second, experience in identifying complex patterns that are presented sequentially may improve the rate of information transmission. Third, with increased rates of presentation, patterns may not be processed singly but in groups, a form of "chunking" information that might reduce the relative importance of individual letters, but emphasize the information in larger patterns of stimulation (Kirman, 1973). Such factors may be responsible for the success of some tactual communication systems that rely on the processing of information from several fingers, such as Tadoma. Finally, the present study showed that performance improved and that the effect of complexity was reduced when patterns were presented to fingers on opposite hands, suggesting that attentional limitations might be partially avoided by presenting patterns bilaterally, a suggestion made previously by Craig (1985a).

\section{REFERENCES}

Bliss, J. C., Crane, H. D., Link, S. W., Townsend, J. T. (1966). Tactile perception of sequentially presented spatial patterns. Perception \& Psychophysics, 1, 125-130.

Bliss, J. C., Katcher, M. H., Rogers, C. H., \& Shepard, R. P. (1970). Optical-to-tactile image conversion for the blind. IEEE Transactions on Man-Machine Systems, MMS-11, 58-64.

BLISS, J. C., \& LiNvilL, J. G. (1966). A direct translation reading aid: Reading alphabetic shapes tactually. In R. Dufton (Ed.), Proceedings of the international conference on sensory devices for the blind (pp. 389-407). London: Arrowsmith.

Cholewiak, R. W., Craig, J. C. (1984). Vibrotactile pattem recognition and discrimination at several body sites. Perception \& Psychophysics, 35, 503-514.

Craig, J. C. (1976). Vibrotactile letter recognition: The effects of a masking stimulus. Perception \& Psychophysics, 20, 317- 326.

CraIG, J. C. (1978). Vibrotactile pattern recognition and masking. In G. Gordon (Ed.), Active touch - the mechanism of recognition of objects by manipulation: A multi-disciplinary approach (pp. 229-242). Oxford: Permagon.

Craig, J. C. (1980). Modes of vibrotactile pattern perception. Journal of Experimental Psychology: Human Perception \& Performance, 6 , $151-166$.

Craig, J. C. (1982). Vibrotactile masking: A comparison of energy and pattern maskers. Perception \& Psychophysics, 31, 523-529.

CraIG, J. C. (1983). The role of onset in the perception of sequentially presented vibrotactile patterns. Perception \& Psychophysics, 34, $421-432$.

Craig, J. C. (1985a). Attending to two fingers: Two hands are better than one. Perception \& Psychophysics, 38, 496-511.

CraIG, J. C. (1985b). Tactile pattern perception and its perturbations. Journal of the Acoustical Society of America, 77, 238-246.

Craig, J. C., Evans, P. M. (1987). Vibrotactile masking and the persistence of tactual features. Perception \& Psychophysics, 42, 309-317.

Craig, J. C., Green, B. A., Rhodes, R. R. (1985). Ipsilateral versus bilateral placement of a tactile vocoder display. Journal of the Acoustical Society of America, 77, 1266-1268.

Craig, J. C., \& XU, B. (1990). Temporal order and tactile patterns. Perception \& Psychophysics, 47, 22-34.

Evans, P. M. (1987a). Vibrotactile masking: Persistence, integration, and strength of representation. Unpublished doctoral dissertation, Indiana University.
Evans, P. M. (1987b). Vibrotactile masking: Temporal integration, persistence, and strengths of representations. Perception \& Psychophysics, 42, 515-525.

Evans, P. M., \& CraIG, J. C. (1986). Temporal integration and vibrotactile backward masking. Journal of Experimental Psychology: Human Perception \& Performance, 12, 160-168.

Evans, P. M., \& Craig, J. C. (1991). Tactile attention and the perception of moving tactile stimuli. Perception \& Psychophysics, 49, 355-364.

Geldard, F. A., \& Sherrick, C. E. (1965). Multiple cutaneous stimulation: The discrimination of vibratory paterns. Joumal of the Acoustical Society of America, 37, 797-801.

Gilson, R. D. (1968). Some factors affecting the spatial discrimination of vibrotactile patterns. Perception \& Psychophysics, 3, 131-136.

Gottheil, E. F., Cholewiak, R. W., \& SherRICK, C. E. (1978). The discrimination of vibratory patterns on a tactile matrix. Bulletin of the Psychonomic Society, 11, 21-24.

HORNER, D. T. (1991). The effects of complexity on the perception of vibrotactile patterns. Perception \& Psychophysics, 49, 551-562.

HoRner, D. T., CRAIG, J. C. (1989). A comparison of discrimination and identification of vibrotactile patterns. Perception \& Psychophysics, 45, 21-30.

KaHneman, D. K. (1973). Attention and effort. Englewood Cliffs, NJ: Prentice-Hall.

KirmaN, J. H. (1973). Tactile communication of speech: A review and an analysis. Psychological Bulletin, 80, 54-74.

Klatzky, R. L., Lederman, S. J., \& Metzger, V. A. (1985). Identifying objects by touch: An "expert system." Perception \& Psychophysics, 37, 299-302.

KNOWLEs, W. B. (1963). Operator loading tasks. Human Factors, 5, 151-161.

LAPPIN, J. S., \& Foulke, E. (1973). Expanding the tactual field of view. Perception \& Psychophysics, 14, 237-241.

MCFADDEN, D. (1970). Three computational versions of proportion correct for use in forced-choice experiments. Perception \& Psychophysics, 8, 336-342.

MORAY, N. (1967). Where is capacity limited? A survey and a model. Acta Psychologica, 27, 84-92.

Navon, D. Gopher, D. (1979). On the economy of the human processing system. Psychological Review, 86, 215-255.

Nolan, C. Y., \& Kederis, C. J. (1969). Perceptual factors in braille word recognition. New York: American Foundation for the Blind.

Norman, D. A., Bobrow, D. G. (1975). On data-limited and resource-limited processes. Cognitive Psychology, 7, 44-64.

PoSNER, M. I. (1978). Chronometric explorations of mind. Hillsdale, NJ: Erlbaum.

PoSNer, M. I. (1982). Cumulative development of attentional theory. American Psychologist, 37, 168-179.

Posner, M. I., \& Mrtchell, R. F. (1967). Chronometric analysis of classification. Psychological Review, 74, 392-409.

Proctor, R. W. (1981). A unified theory for matching task phenomena. Psychological Review, 88, 291-326.

Reed, C. M., Durlach, N. J., Braida, L. D., \& Schultz, M. C. (1982). Analytic study of the Tadoma method: Identification of consonants and vowels by an experienced Tadoma user. Journal of Speech \& Hearing Research, 25, 108-116.

Robinson, J. S., Brown, L. T., Hayes, W. H. (1964). Test of effects of past experience on perception. Perceptual \& Motor Skills, 18, 953-956.

Shiffrin, R. M., Craig, J. C., \& Cohen, E. (1973). On the degree of attention and capacity limitation in tactile processing. Perception \& Psychophysics, 13, 328-336.

Wickens, C. D. (1984). Processing resources in attention. In R. Parasuraman \& D. R. Davies (Eds.), Varieties of antention (pp. 63102). New York: Academic Press.

(Manuscript received August 12, 1991; revision accepted for publication February 25, 1992). 